\title{
A mathematical formulation for the cell-cycle model in somitogenesis: analysis, parameter constraints and numerical solutions
}

\author{
D. McInerney, S. SChnell $\dagger * *$, R. E. BAKer And P. K. Maini $\ddagger$ \\ Centre for Mathematical Biology, Mathematical Institute, 24-29 St Giles', Oxford \\ OX1 3LB, UK
}

[Received on 14 June 2002; revised on 26 January 2004]

\begin{abstract}
In this work we present an analysis, supported by numerical simulations, of the formulation of the cell-cycle model for somitogenesis proposed in Collier et al. (J. Theor. Biol. 207 (2000), 305-316). The analysis indicates that by introducing appropriate parameter constraints on model parameters the cell-cycle mechanism can indeed give rise to the periodic pattern of somites observed in normal embryos. The analysis also provides a greater understanding of the signalling process controlling somite formation and allows us to understand which parameters influence somite length.
\end{abstract}

Keywords: somitogenesis; cell-cycle; signalling; finite element; pattern formation.

This article is dedicated to Dr R. W. Hiorns in recognition of his work as the editor of IMA Journal of Mathematics Applied in Medicine and Biology.

\section{Introduction}

For all but the simplest multicellular development a common mode of patterning involves the laying down of structures behind a moving front. One such example is the formation of somites.

Somites are spheres of cells formed sequentially on each side of the anterior-posterior (AP) axis of a developing embryo by the successive segmentation of the presomitic mesoderm (PSM). A somite is formed by the condensation of groups of mesenchymal cells which become polarized and form a three-dimensional epithelium. They are transitory structures that underlie the segmental organization of the vertebrate body that is maintained into adulthood with the formation of the vertebral column and govern the segmental organization of peripheral spinal nerves, backbone and vertebrae, axial muscles, thorax, and the metameric distribution of early blood vessels. Genetic or environmental factors can disturb somitogenesis, and there are many clinical conditions which occur as a result (see, for recent reviews in the subject, Gossler \& Hrabě de Angelis, 1998; Stickney et al., 2000; Stockdale et al., 2000; Schnell et al., 2000; Saga \& Takeda, 2001, and references therein).

\footnotetext{
$\dagger^{\dagger}$ Also at: Christ Church, Oxford OX1 1DP, UK, Email: schnell@maths.ox.ac.uk

‡Email: maini@maths.ox.ac.uk

${ }^{*}$ Corresponding author
} 
Over the past three decades, somitogenesis has been studied in detail. However, despite considerable experimental work, the mechanism of this aggregation of cells into somites and the specification of where and how many somites will eventually develop is still a major unresolved problem in developmental biology. Several theoretical and mathematical models have been proposed to address some of these questions. Recently, we have reviewed a large number of theoretical models for somitogenesis and have found that although some of these successfully account for certain aspects of somitogenesis, they fail to explain, or even contradict, other observations (Schnell \& Maini, 2000; Schnell et al., 2000; Baker et al., 2003).

We have addressed (Collier et al., 2000) some of the problems concerning somite formation, by developing a mathematical formulation of the cell-cycle model for somitogenesis proposed by Stern and co-workers (Stern et al., 1988; Primmett et al., 1989). The cell-cycle model appears to be consistent with many experimental observations as it incorporates several known aspects of somitogenesis. For example, it can account for the periodic abnormalities observed in somite formation after a single heat shock treatment (Cooke, 1978; Primmett et al., 1988); the cell autonomous character of somite formation in PSM explants, the grafting experiments showing that presumptive somites -I to $-\mathrm{V}$ (corresponding to one cell-cycle before somite formation) are fixed with respect to their AP polarity, boundary transplantation experiments reversing the AP axis (Dubrulle et al., 2001), and it provides a framework which allows investigation of the movement properties of the cells in the presomitic mesoderm as they aggregate into a somite (readers can consult Collier et al., 2000; Schnell et al., 2002, for more details). The mathematical formulation developed consists of two coupled partial differential equations, whose nonlinear coupling gives rise to a regular pulse-like signal. We have also presented an extension of the cellcycle model (Baker et al., 2003) to take account of the existence of a determination front whose position in the PSM is controlled by FGF-8 signalling (Dubrulle et al., 2001; Sawada et al., 2001), and which controls the ability of cells to become competent to segment.

The mathematical model has enabled us to compute the outcome of the nonlinear interactions arising from the word model of Stern and co-workers. In previous papers, we have verified the role of the cell-cycle as a possible segmentation clock, analysed the effects of heat shock in somite formation (Collier et al., 2000), included cell movement for the version of the model in one spatial dimension (Schnell et al., 2002) and analysed the effects of the local application or inhibition of FGF-8 by implantation of a bead soaked in FGF-8 in or next to the PSM (Baker et al., 2003). We have also solved the model equations numerically using the finite element method and compared the model behaviour with experimental observations.

In the present paper, we study in more detail the mathematical model proposed in our previous papers (Collier et al., 2000; Schnell et al., 2002). After an exposition of the descriptive model, we introduce its assumptions and a mathematical formulation in Section 2. A phase plane study of the model equations gives an insight into the behaviour of the kinetics allowing us to derive bounds on parameter values of the model to ensure a regular pulse-like signal which coordinates the differentiation of cells located anterior in the PSM to segment into a discrete somite (Section 3). The behaviour of the signalling process controlling somite formation is analysed in Section 4, where an analytical approximation of the signalling chemical profile is derived. We present our 
numerical solutions followed by a comparison between the analytical and numerical results (Section 5). In Section 6, we discuss our analysis of the model equations which enables us to place constraints on model parameters, and to understand the signalling process and how various parameters influence somite length. Finally, we present a brief conclusion in Section 7.

\section{The cell-cycle model}

The cell-cycle model proposes the cell-cycle as a possible segmentation clock based on the following observations: (1) the existence of discrete regions of cell synchrony in the PSM; (2) a cell-cycle duration of nine hours, which corresponds to the development of six to seven somites in the chick embryo; (3) periodic segmental anomalies (such as a small somite followed by a large somite) caused by heat shock experiments or drugs inhibiting cell-cycle progression (Stern et al., 1988; Primmett et al., 1988, 1989).

According to the cell-cycle model, cells destined to form somites leave Hensen's node (see Fig. 1) strictly in the order in which they were derived from founder cells in the node, and they remain in that order. Hence there is some degree of cell-cycle synchrony of somite cells at the same level and these cells will be arranged along the embryonic axis in the same order as they are positioned in their cell-cycles: older cells further anterior than younger ones (Stern et al., 1992).

The formation of a somite is explained by assuming that there are two successive time points $P_{1}$ and $P_{2}$ in the cell-cycle of the mesodermal cells destined to form a somite. These points are assumed to be 90 minutes apart in the chick embryo, near the time of the second last mitotic division before segmentation (Fig. 1). This determination phase comprises about one-seventh of the total cell-cycle time. Somite cells recognize the $P_{1-}$ $P_{2}$ time window. As the cell-cycles of all cells are not perfectly synchronized some cells will reach point $P_{2}$ in their cell-cycle before others. These cells produce a signal to which cells situated between $P_{1}$ and $P_{2}$ respond (Region II in Fig. 1). Cells between $P_{1}$ and $P_{2}$ later increase their adhesion to each other shortly before segmentation and clump together. The resulting group of cells undergoes segmentation one cell-cycle after the $P_{1}-P_{2}$ time window. After the $P_{1}-P_{2}$ window, cells become refractory (unresponsive) to the signal and/or unable to signal (Region III in Fig. 1).

The model proposes that heat shock temporarily blocks the cell-cycle, so altering the number of cells that become adhesive together. Such an alteration would occur once in the cell-cycle of each cell posterior to the $P_{1}-P_{2}$ time window, accounting for the repetitive anomalies resulting from heat shock.

\subsection{Mathematical formulation}

A mathematical formalisation of the cell-cycle model has enabled us to explore more closely the interactions of the underlying processes hypothesized in this model and to make further experimentally testable predictions. We begin by considering a coupled pair of nonlinear partial differential equations proposed by Collier et al. (2000) which models the appropriate spatio-temporal cell signalling suggested by the biological model.

The key feature of the Stern and co-workers' model is that segmentation arises as a result of some local mechanism which is regulated by time points in the cell-cycle of the 


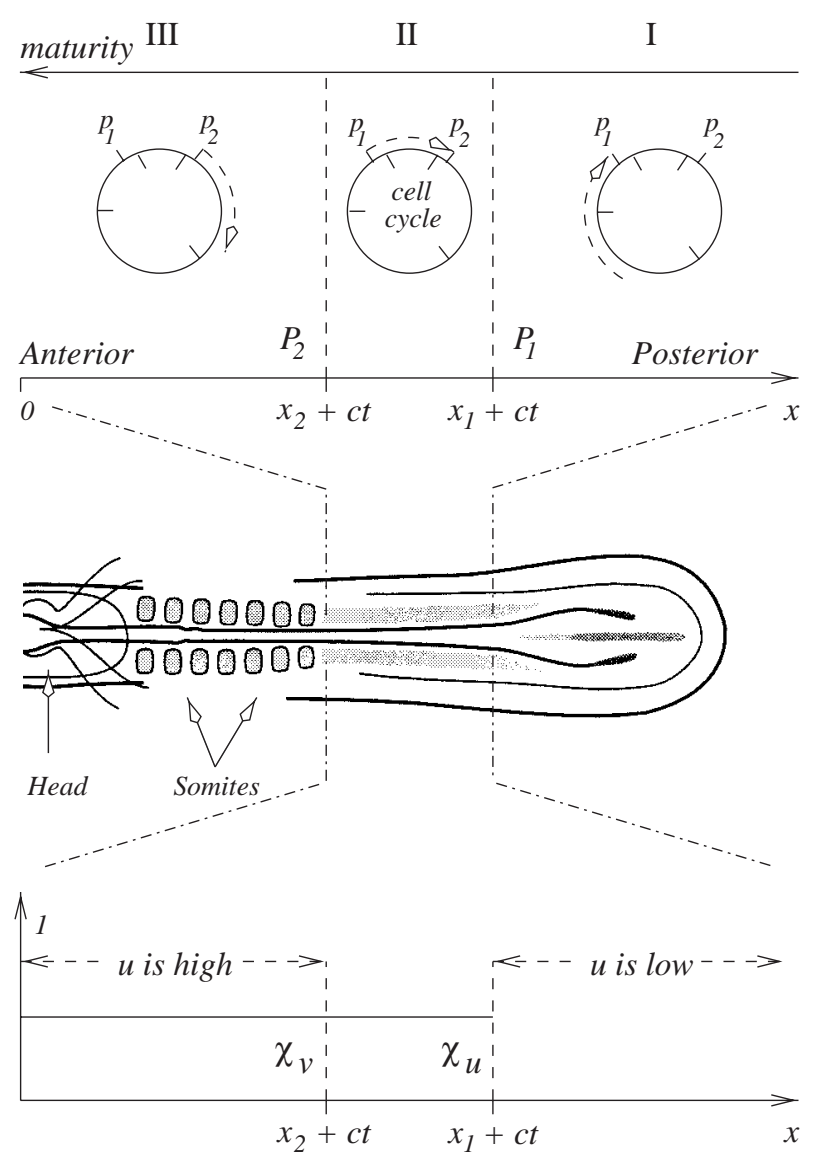

FIG. 1. Diagrammatic representation of the cell-cycle model illustrating the two time points $P_{1}$ and $P_{2}$ and the three key stages within the model. Cells at the posterior-most margin of the PSM (Region I) are at a younger developmental age than those undergoing somitogenesis. As these cells mature, they become capable (Region II) of responding to the signalling molecule $v$, which is secreted by cells at $P_{2}$. As they mature to the next stage (Region III) they begin to form somites. In Region III they are no longer able to respond to or emit a signal. A diagrammatic representation of the mathematical formulation of Collier et al. (2000) is shown below the sketch depicting the fully formed somites. The Heaviside functions $\chi_{u}=H\left(x_{1}+c t-x\right)$ and $\chi_{v}=H\left(x_{2}+c t-x\right)$ are shown along with the regions where the somitic potential factor $u$ is, respectively, high $\left(x<x_{2}+c t\right)$ and low $\left(x>x_{1}+c t\right)$. For a full description of the model kinetics see Section 2.1.

cells destined to form a particular somite. After specification, these cells adhere to each other. All cells of a potential somite must adhere at about the same time, resulting in a distinct somite. In addition to the assumptions mentioned previously, in formulating the mathematical model Collier et al. (2000) further assumed that: (1) although segmentation begins at the anterior end of the embryo and moves towards the posterior, for convenience the axis is taken fixed with respect to the cells so that the pattern (including Hensen's node and the PSM) can be considered to be moving down the rostrocaudal axis. The length of the PSM is constant, and the pattern moves with constant speed $c\left(c \approx 2.0 \times 10^{-8} \mathrm{~ms}^{-1}\right.$ in the trunk region of the chick); (2) the signal emitted by cells when they reach point $P_{2}$ 
is like a pulse. The signalling molecule is short-lived and diffuses rapidly, this is a key assumption. Rapid diffusion is required to ensure that only cells whose cell-cycle is at a point between $P_{1}$ and $P_{2}$ will respond to this signal, if the diffusion coefficient is not sufficiently large, then somite size will be determined by the extent to which the signal spreads; and (3) somites are being formed continually; the beginning or end of this process is not considered.

Although the development of biological pattern and form during embryogenesis is a three-dimensional process, the model is developed in one spatial dimension (head-to-tail axis). This simplification enables us to study in detail the interaction of the nonlinear model kinetics and allows us to investigate many of the key patterning processes, which are known to act primarily along this axis. Additionally, it has been assumed that it is sufficient to focus on one side of the embryo. This is supported by evidence which suggests that there is no signalling between the two parallel axes of the PSM (Keynes \& Stern, 1988; Veini \& Bellairs, 1986).

We let $x$ denote distance down the head-to-tail axis of the embryo, where the origin is taken to be fixed with respect to a given somite (a given cell has a fixed $x$-coordinate), and $t$ denotes time. It is proposed that a key step in determining a cell's fate as part of a somite is exposure to a somitic factor of concentration $u(x, t)$. This somitic factor might be a transcription factor or a precursor of an adhesion molecule. Cells which have a high concentration of $u$ are termed somitic, while those with a low concentration are termed non-somitic. The somitic factor is produced in response to a diffusive signalling molecule, $v(x, t)$, the other key component in the model.

It is assumed that cells passing point $P_{2}$ produce $v$, while cells which lie between points $P_{1}$ and $P_{2}$ respond to $v$ by producing $u$. In Fig. 1 it can be seen that the cells which reach point $P_{1}$ in their cell-cycle at a given time $t$ are at position $x_{1}+c t$ on the axis, while those which reach $P_{2}$ are at position $x_{2}+c t$. Cells positioned anterior to $x_{1}+c t$ have passed point $P_{1}$ in their cell-cycle and so can produce $u$, and the signalling molecule $v$ is produced when cells pass $P_{2}\left(x_{2}+c t\right)$. The model equations are

$$
\begin{aligned}
& \frac{\partial u}{\partial t}=f(u, v), \\
& \frac{\partial v}{\partial t}=g(u, v)+D \frac{\partial^{2} v}{\partial x^{2}} .
\end{aligned}
$$

The forcing functions are given by

$$
f(u, v)=\frac{(u+\mu v)^{2}}{\gamma+\rho u^{2}} \chi_{u}-v u, \quad g(u, v)=\frac{\kappa}{\epsilon+u} \chi_{v}-\lambda v,
$$

where

$$
\chi_{u}(x, t)=H\left(c t-x+x_{1}\right), \quad \chi_{v}(x, t)=H\left(c t-x+x_{2}\right) .
$$

The Heaviside function, $H$, is of the general form $H(\alpha-x)$, where

$$
H(\alpha-x)= \begin{cases}1 & \text { if } x \leqslant \alpha \\ 0 & \text { if } x>\alpha\end{cases}
$$

and $\mu, \gamma, \rho, v, \kappa, \epsilon, \lambda, D, c, x_{1}, x_{2}$ are positive constants. 
The switch behaviour occurring in the kinetics of the somitic factor $u$ is captured in the above mathematical formulation by the right-hand side of (1a). The kinetics for $u$ are chosen so that $u$ can undergo a sudden change from a low to a high concentration. The term $(u+\mu v)^{2} /\left(\gamma+\rho u^{2}\right)$ describes auto-catalytic production of $u$, activation of $u$ by $v$ through the term $\mu v$, and, for large $u$, saturation. Furthermore, $u$ is linearly degraded in proportion to its concentration by the term $-v u$. For $(1 \mathrm{~b})$, the nonlinear term $\frac{1}{\epsilon+u}$ models negative feedback by $u$ on the production of $v$, i.e. an increase in $u$ decreases the production of $v$, which results in a reduction in $u$ production (1a). For $\epsilon \ll 1, v$ increases rapidly for $u \ll 1$, i.e. there is an increase in $v$ in non-somitic cells. In addition, $v$ degrades linearly and diffuses, with diffusion coefficient $D$.

Notice that the nonlinear component in (1a) does not become active until $x \leqslant x_{1}+c t$. In Fig. 1, it can be appreciated that points to the left of $x_{1}+c t$ correspond to cells which have passed point $P_{1}$ in their cell-cycle. This is consistent with the requirement that cells whose cell-cycle lies between points $P_{1}$ and $P_{2}$ (which at time $t$ are at positions $x_{1}+c t$ and $x_{2}+c t$ respectively) can respond to a pulse in $v$ and it is achieved in the equation by multiplying the nonlinear component of kinetics for the somitic factor (1a) by the Heaviside function $\chi_{u}(x, t)$ (see Fig. 1). In (1b) the nonlinear term satisfies the model requirement that only cells which have not been specified as somitic, cells with low concentration of $u$, can produce the signalling molecule $v$ while those that have been specified as somitic, cells with high concentration of $u$, cannot. Furthermore, we must ensure that signalling does not begin until $x \leqslant x_{2}+c t$, points to the left of $x_{2}+c t$ correspond to cells which have passed point $P_{2}$ in their cell-cycle, as cells must pass point $P_{2}$ before they can signal. This is captured in the cell signalling equation (1b) by multiplying the nonlinear component of the kinetics by the Heaviside term $\chi_{v}(x, t)$.

The model is envisioned to work as follows: at time $t=0, u \approx 0$ for all points $x>x_{2}$ and $u \approx 1$ for points $x<x_{2}$, while the level of signalling molecule is $\mathrm{O}(1)$ throughout the domain. As time increases the Heaviside functions $\chi_{u}$ and $\chi_{v}$ (see Fig. 1) move to the right at speed $c$. Points where $u \approx 0$, which were initially to the right of $\chi_{v}$, quickly become part of the region where $\chi_{v}$ is active. These low values of $u$ activate $v$ production leading to a pulse in $v$ centred at $x_{2}+c t$. At some time $t=\tilde{t}$ (shortly after $t=0$ ) the increase in $v$ activates $u$ between points $x=x_{2}$ and $x=x_{1}+c \tilde{t}$. As $u$ rises it inhibits $v$ production, and the pulse-like distribution of $v$ quickly falls returning to $v \sim 1$. As $v$ falls the rise in $u$ slows. A short time after $t=\tilde{t}, u \approx 1$ for all points between $x_{2}$ and $x_{1}+c \tilde{t}$. In this way, the region $x_{2}<x<x_{1}+c \tilde{t}$ is specified as somitic. The process begins again when the jump in the Heaviside function $\chi_{v}$ reaches point $x_{1}+c \tilde{t}$. Points where $u \approx 0$ become part of the region where $\chi_{v}$ is active. These low values of $u$ activate $v$ production leading to a second pulse in $v$.

From our description above it is clear that there is a small delay between signalling and specification. Hence, cells gain the potential to form a somite a short time after the signal is emitted. Although cells passing point $P_{2}$ at time $t=0$ start producing $v$ almost immediately, the pulse-like signal does not rise and fall in an instant. It is not until $t=$ $\tilde{t}$ that $v$ is sufficiently high to activate $u$. We will discuss this point in the next section (Section 3) by studying the parameter constraints and the threshold level of signalling molecule required to activate this periodic signalling process. 
Boundary conditions. The embryonic axis is taken fixed with respect to the cells, the node and segmental plates move down it at a constant rate $c$. We consider

$$
0 \leqslant x \leqslant d(t), \quad t \geqslant 0,
$$

where $d(t)$ is the position of a point which moves down the embryonic axis at constant speed $c$. For points on the axis far to the right of $x_{2}+c t$, that is $x \gg x_{2}+c t, u$ and $v$ tend to zero, while for points to the left, $u$ and $v$ tend to some fixed value. This leads to the following boundary conditions:

$$
\begin{aligned}
u, v \rightarrow 0 \text { as } x-\left\{x_{2}+c t\right\} & \rightarrow+\infty, \\
u, v \text { are bounded as } x-\left\{x_{2}+c t\right\} & \rightarrow-\infty .
\end{aligned}
$$

In our numerical simulations (Collier et al., 2000; Schnell et al., 2002), the equations have been solved on a closed bounded interval of $\mathbb{R}$, which we denote by $\Omega$; typically $\Omega=$ $[-10,10]$, which is large enough to ensure that conditions at the boundary do not affect the patterning process. As an approximation to these infinite domain boundary conditions, (1a) is subject to zero flux boundary conditions at $x=-10$ and $x=10$.

Non-dimensionalization. The equations are non-dimensionalized to allow an absolute measure of the quantities involved independent of units of measurement and to reduce the number of parameters. We choose

$$
t=\frac{\hat{t}}{\lambda}, \quad x=\left(x_{1}-x_{2}\right) \hat{x}, \quad u=\frac{\hat{u}}{\nu \rho}, \quad v=\frac{\kappa \nu \rho}{\lambda} \hat{v} .
$$

In the non-dimensional variables $\hat{x}, \hat{t}, \hat{u}$ and $\hat{v}$, the distance between $x_{1}+c t$ and $x_{2}+$ $c t$ equals 1 . The process occurs on a time scale in which $u$ rises from near zero to its equilibrium state; in this equilibrium both $\hat{u}$ and $\hat{v}$ are $O(1)$. Substituting (4) into (1a) and (1b) and re-arranging, the following dimensionless parameters can be defined:

$$
\begin{aligned}
& \hat{\mu}=\mu \kappa v^{2} \rho^{2} \lambda^{-1}, \quad \hat{\gamma}=\gamma \lambda v \rho, \quad \hat{\kappa}=\frac{\lambda}{v}, \quad \hat{\epsilon}=\epsilon \nu \rho, \\
& \hat{D}=\frac{D}{\lambda\left(x_{1}-x_{2}\right)^{2}}, \quad \hat{c}=\frac{c}{v\left(x_{1}-x_{2}\right)}, \quad \hat{x}_{1}=\frac{x_{1}}{x_{1}-x_{2}}, \quad \hat{x}_{2}=\frac{x_{2}}{x_{1}-x_{2}} .
\end{aligned}
$$

This yields the following non-dimensional model, where the hats have been dropped for notational convenience:

$$
\begin{aligned}
& \frac{\partial u}{\partial t}=f(u, v), \\
& \frac{\partial v}{\partial t}=g(u, v)+D \frac{\partial^{2} v}{\partial x^{2}},
\end{aligned}
$$

with the forcing functions given by

$$
f(u, v)=\frac{(u+\mu v)^{2}}{\gamma+\kappa u^{2}} \chi_{u}(x, t)-\frac{u}{\kappa}, \quad g(u, v)=\frac{\chi_{v}(x, t)}{\epsilon+u}-v,
$$


and

$$
\chi_{u}(x, t)=H\left(c t-x+x_{1}\right), \quad \chi_{v}(x, t)=H\left(c t-x+x_{2}\right), \quad x_{1}-x_{2}=1 .
$$

The main changes in $u$ and $v$ occur in region II (see Fig. 1) and the part of regions I and III close to II. In the next section the behaviour of the model in regions I, II and III as labelled in Fig. 1 is explored.

\section{Phase plane study of the model equations}

In a previous paper (Collier et al., 2000) we stated the constraints necessary on the model parameter values to ensure that the cells of a potential somite adhere together, resulting in the segmentation of a discrete somite. Here, we present full details of the derivation of these constraints. The model equations behave differently in each of the three regions along the embryonic axis, as illustrated in Fig. 1.

I Cells posterior to $P_{1}, x>x_{1}+c t$, can neither respond to nor emit a signal. For these cells, both $u$ and $v$ are approximately zero.

II Cells between $P_{1}$ and $P_{2}, x_{2}+c t<x \leqslant x_{1}+c t$, can respond to a signal but are unable to emit one. These cells respond to a high level of $v$ by producing $u$. As $u$ increases, $v$ falls, giving rise to a pulse-like signal in $v$.

III Cells anterior to $P_{2}, x \leqslant x_{2}+c t$, can respond to and emit a signal. Here, a high level of $u$ inhibits production of $v$.

We now perform a phase plane analysis of the model equations in each of the regions labelled in Fig. 1, neglecting the effect of diffusion. How diffusion affects the patterning process is studied in detail in Section 4. As always, we must keep in mind the model requirements as described in Section 2.1, in particular, the constraints placed on the behaviour of the forcing functions. In Section 3.5 a summary of the bounds derived on the parameters is presented.

\subsection{The behaviour of the model in region I}

In the region $x>x_{1}+c t$, the model equations (5a) and (5b) simplify to

$$
\begin{aligned}
& \frac{\partial u}{\partial t}=-\frac{u}{\kappa}, \\
& \frac{\partial v}{\partial t}=-v .
\end{aligned}
$$

The steady states of $u$ and $v$ are zero. On setting $\kappa \gg 1$, we ensure that $v$ decays rapidly compared to $u$. 


\subsection{The behaviour of the model in region II}

In this region, $x_{2}+c t<x \leqslant x_{1}+c t$, the model equations are as follows:

$$
\begin{aligned}
& \frac{\partial u}{\partial t}=\frac{(u+\mu v)^{2}}{\gamma+\kappa u^{2}}-\frac{u}{\kappa}, \\
& \frac{\partial v}{\partial t}=-v .
\end{aligned}
$$

In accordance with the model requirements outlined previously, $u$ must change from approximately zero to a stable steady state with positive value. There are two possible cases depending on the values of $\gamma$ and $\kappa$. Standard linear stability analysis reveals that only for $\gamma<\frac{\kappa}{4}$ does there exist a stable steady state with positive $u$. For this case, there are three non-negative steady states: $(0,0),\left(\tilde{u}_{1}, 0\right)$ and $\left(\tilde{u}_{2}, 0\right)$, with

$$
\tilde{u}_{1,2}=\frac{1}{2}\left(1 \pm \sqrt{1-\frac{4 \gamma}{\kappa}}\right) .
$$

In system (9a) and $(9 \mathrm{~b}),(0,0)$ and $\left(\tilde{u}_{2}, 0\right)$ are linearly stable and $\left(\tilde{u}_{1}, 0\right)$ is a saddle point. A key requirement in the model formulation is that $u$ should change from $u \approx 0$ to $u \sim \mathrm{O}(1)$ in response to a rise in $v$. If $v$ is sufficiently high then $u$ will change from approximately zero to the stable steady state $\tilde{u}_{2}$.

In Fig. 2 we see that the stable manifold at the critical point $\left(\tilde{u}_{1}, 0\right)$ lies on the separatrix, $(\bar{u}, \bar{v})$, that divides the positive quadrant of the phase plane into two regions: trajectories to the left of the separatrix converge to the zero steady state, while those to the right tend to the steady state $\left(\tilde{u}_{2}, 0\right)$. The separatrix must cross the $v$-axis at some point $(0, \theta)$ (see Fig. 2). If not, then $\bar{v} \rightarrow \infty$ while $\bar{u}>0$ as $t \rightarrow-\infty$. From (9a) and (9b) we see that since $\bar{v}$ is unbounded as $t \rightarrow-\infty$, there exists a time, $\tilde{t} \in \mathbb{R}$, where $\bar{v}$ is sufficiently large to make $\partial u / \partial t \geqslant 1$ for all $t<\tilde{t}$. However, this would imply that $\bar{u} \rightarrow-\infty$ as $t \rightarrow-\infty$, contradicting the requirement that $\bar{u}>0$ for all $t \in \mathbb{R}$. Trajectories starting above $(0, \theta)$ converge to the steady state $\left(\tilde{u}_{2}, 0\right)$. In this way $\theta$ can be viewed as the minimum level (threshold level) of $v$ required to activate $u$ production.

Region II will be affected by diffusion of $v$ so $u$ should remain low at points which have not responded to a pulse of $v$, even those for which $v \approx 1$, i.e. $u<\tilde{u}_{1} \sim \frac{\gamma}{\kappa} \ll 1$, when $v \approx 1$, so ( 9 a) becomes

$$
\frac{\partial u}{\partial t}=\frac{(u+\mu v)^{2}}{\gamma+\kappa u^{2}}-\frac{u}{\kappa} \approx \frac{(u+\mu)^{2}}{\gamma}-\frac{u}{\kappa} .
$$

Equation (11) has positive steady states if

$$
\mu<\frac{\gamma}{4 \kappa} \Rightarrow \frac{\gamma}{4 \mu \kappa}>1,
$$

otherwise $\frac{\partial u}{\partial t}>0 \forall u \geqslant 0$. We define

$$
\theta_{l}:=\frac{\gamma}{4 \mu \kappa},
$$




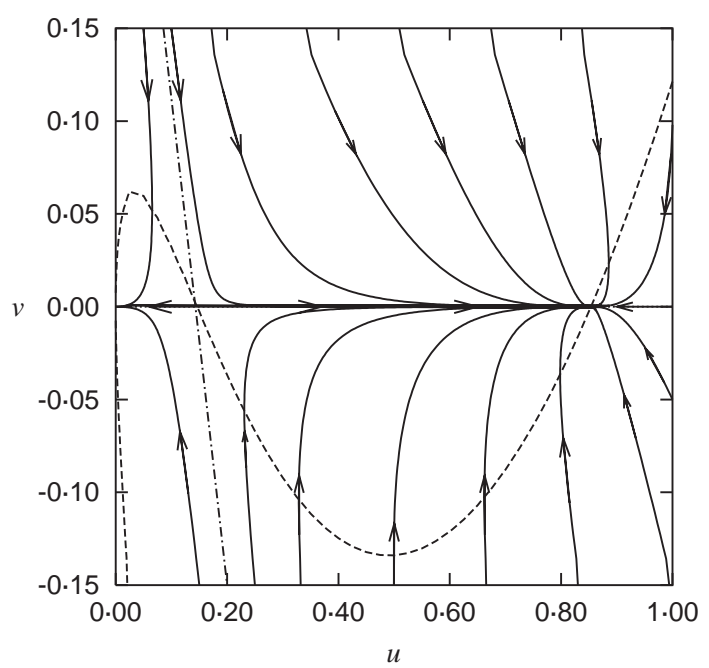

FIG. 2. Phase plane for region II for the case $\gamma<\frac{\kappa}{4}$. The phase plane is divided into two regions by the separatrix (dotted-dashed curve). Parameters are chosen such that the intersections of the $u$ null cline (dashed curve) with the $v$ null cline $(v=0)$ yields three non-negative steady states. Note that if $v$ is sufficiently high $u$ will change from approximately zero to a stable steady state of positive value. Parameter values are: $\mu=0.5, \kappa=2$ and $\gamma=0 \cdot 25$.

where $\theta_{l}$ can be viewed as a lower bound for the threshold $\theta$ that must be exceeded by $v$ in order to activate $u$ at points between $x_{2}+c t$ and $x_{1}+c t$. Alternatively, if $\mu \kappa \ll \gamma$ then the inequality (12) is satisfied. Choosing $\mu \kappa$ and $\gamma$ such that $\mu \kappa / \gamma \leqslant \mathrm{O}(1)$, the condition is violated, and as a result a level of $v \approx 1$ will activate $u$ production.

\subsection{The behaviour of the model in region III}

In this region, $x \leqslant x_{2}+c t$ and hence the model equations become

$$
\begin{aligned}
& \frac{\partial u}{\partial t}=\frac{(u+\mu v)^{2}}{\gamma+\kappa u^{2}}-\frac{u}{\kappa}, \\
& \frac{\partial v}{\partial t}=\frac{1}{\epsilon+u}-v .
\end{aligned}
$$

These equations yield either one or three positive steady states. The null clines are as follows:

$$
\begin{aligned}
& v=\Psi(u):=\frac{1}{\mu}\left(-u \pm \sqrt{\frac{u\left(\gamma+\kappa u^{2}\right)}{\kappa}}\right), \\
& v=\lambda(u):=\frac{1}{\epsilon+u} .
\end{aligned}
$$

Parameters must be chosen to ensure that a stable steady state with a high level of $u$ is the only stable steady state in the region (according to our model, cells which have 
responded to a pulse in signalling molecule have a high concentration of $u$ ). How the cells react to the sudden increase in $v$ is studied by looking at trajectories in the phase plane. It is found that to ensure the existence of exactly one positive steady state in this region the $v$ null cline must lie above the local maximum of the $u$ null cline.

On choosing $\gamma \ll \frac{\kappa}{4}$, the unstable steady state $\tilde{u}_{1}$ can be approximated as follows:

$$
\tilde{u}_{1}=\frac{1}{2}\left(1-\sqrt{1-\frac{4 \gamma}{\kappa}}\right) \sim \frac{\gamma}{\kappa}+\mathrm{O}\left\{\left(\frac{\gamma}{\kappa}\right)^{2}\right\} .
$$

In Fig. 2 we see that the $u$ null cline has a local maximum at $\left(u_{m}, v_{m}\right)$, with $0<u_{m}<$ $\tilde{u}_{1} \sim \frac{\gamma}{\kappa}$. Expanding $u$ in terms of a small parameter $\delta$, where $\delta=\frac{\gamma}{\kappa} \ll 1$, we obtain the leading order problem for $u_{0}$ :

$$
9 u_{0}^{4}-4 u_{0}^{3}=0 \quad \Rightarrow u_{0}=0, \frac{4}{9} .
$$

Since $u_{m}<\frac{\gamma}{\kappa} \ll 1$, we choose $u_{0}=0$. Similarly, on collecting the higher order terms we find that $u_{1}=\frac{1}{4}$. In this way, we obtain the following estimates for $\left(u_{m}, v_{m}\right)$ :

$$
u_{m}=\frac{\gamma}{4 \kappa}+\mathrm{O}\left(\delta^{2}\right), \quad v_{m}=\frac{\gamma}{4 \mu \kappa}+\mathrm{O}\left(\delta^{2}\right) .
$$

The phase plane for the case $\gamma<\frac{\kappa}{4}$ is shown in Fig. 3. To ensure the existence of exactly one positive stable steady state in this region the $v$ null cline must lie above the local maximum of the $u$ null cline (15a), and so, to a low order of approximation

$$
\frac{1}{\epsilon+\frac{\gamma}{4 \kappa}}>\frac{\gamma}{4 \mu \kappa} \text {. }
$$

At time $t=\tilde{t}$, just before a signal is emitted, $u \approx 0$ at $x=x_{2}+c \tilde{t}$, and hence $v$ increases rapidly, so (14b) becomes

$$
\frac{\partial v}{\partial t}=\frac{1}{\epsilon}-v
$$

Since, from this equation, $v$ cannot rise above $\frac{1}{\epsilon}$, the threshold level $\theta$, i.e. the minimum level of $v$ required to activate $u$ production, must be significantly lower than $\frac{1}{\epsilon}$ and above the local maximum of the $u$ null cline. Therefore, the parameters are chosen such that

$$
\frac{1}{\epsilon} \gg \theta>v_{m} \sim \frac{\gamma}{4 \mu \kappa} \Rightarrow \frac{\gamma \epsilon}{4 \mu \kappa} \ll 1
$$

As seen in the previous section, for points between $x_{2}+c t<x \leqslant x_{1}+c t$ which have already responded to a signal, $u \sim \tilde{u}_{2} \approx 1$. These points must not trigger another signal when the source term $(\epsilon+u)^{-1}$ becomes active: only points where $u \approx 0$ should lead to a signal. Choosing

$$
\epsilon \ll \tilde{u}_{2} \approx 1
$$

ensures that the source term $(\epsilon+u)^{-1}$ becomes large for points where $u \ll 1$. Hence $v$ increases rapidly for $u \ll 1$. 


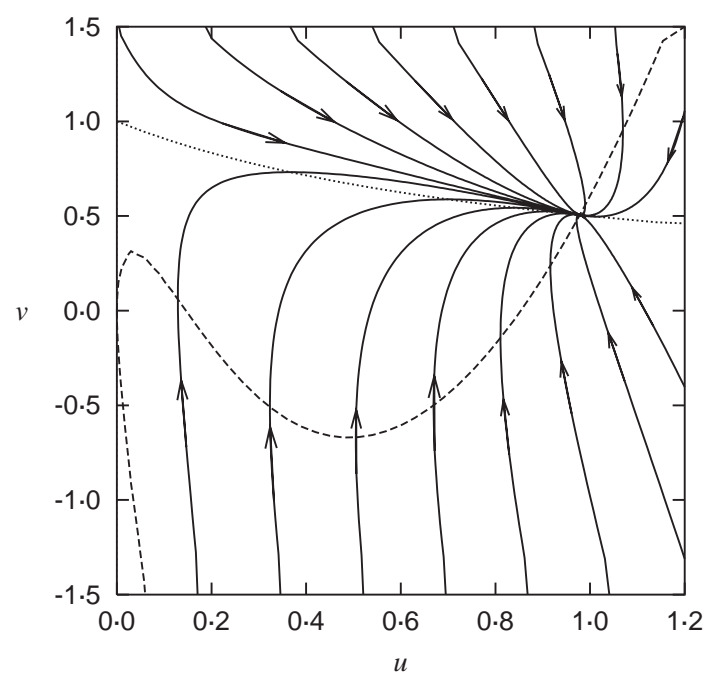

FIG. 3. Phase plane for region III. Parameters are chosen such that the intersection of the $u$ null cline (dashed curve) with the $v$ null cline (dotted curve) yields one positive steady state, $\tilde{u}$. Note that all trajectories converge to the steady state. Parameter values are: $\mu=0 \cdot 1, \kappa=2, \gamma=0.25$ and $\epsilon=1$.

\subsection{Effect of diffusion}

A requirement of the model is that the posterior boundary of a somite is determined by the point $P_{1}$ (to be consistent with the cell-cycle model) in the cell-cycle rather than by diffusion of the signalling molecule, $v$, i.e. if cells fail to respond to the pulse it is because they have not reached $P_{1}$ in their cell-cycle, not because the signal fails to diffuse far enough. To ensure that lack of diffusion does not prevent cells from gaining the potential to form a somite we take

$$
D \gg 1 \text {. }
$$

In our discussion of the model kinetics we saw how cells gain the potential to form a somite a short time after the signal is emitted. Furthermore, there is a time interval during which specification occurs, i.e. the time when $v$ is above the threshold. To ensure that this interval is small, the parameters are chosen such that the signal does not persist above the threshold for too long. Otherwise, cells which go on to form a somite will become potentially somitic over a long period of time (comparable to the time taken to form a somite).

\subsection{Summary of parameter constraints}

From Sections 3.1 and 3.2 we have the following two parameter constraints:

$$
\kappa \gg 1, \quad \gamma<\frac{\kappa}{4} \text {. }
$$

The first constraint, $\kappa \gg 1$, ensures that $v$ decays rapidly compared to $u$. On imposing the second constraint, $u$ changes from a low to a high level if $v$ is sufficiently high. We set 
$\gamma \ll 1$, to ensure this constraint is satisfied. Furthermore, from (3.2) it can be seen that $\tilde{u}_{2} \approx 1$ for $\gamma \ll 1$.

In region II one of the key model requirements is that $u$ should remain low in the presence of $v \approx 1$. This is expressed by

$$
\theta_{l}>1,
$$

where $\theta_{l}$ is a lower bound for $\theta$, the threshold that must be exceeded by $v$ to activate $u$ production. The parameter constraints in Section 3.3 can be related to the threshold level $\theta$ of signalling molecule via (13). In Fig. 3 we see that to ensure the existence of exactly one positive stable steady state in region III, the $v$ null cline must lie above the local maximum of the $u$ null cline. To a first order approximation, $\theta_{l}(13)$ is equal to the local maximum of the $u$ null cline (18). Hence, expression (19) can be written as follows:

$$
\theta_{l}<\frac{1}{\epsilon+\frac{\gamma}{4 \kappa}} .
$$

Again, using the definition of $\theta_{l}$ (13), the parameter bound (21) can be written as

$$
\theta_{l} \ll \frac{1}{\epsilon} .
$$

Finally, diffusion must be sufficiently high so that all cells gain the potential to form a somite. For each condition, a set of parameters which violates that condition has been considered and the resulting numerical solution plotted (see Section 6).

\subsection{Initial conditions}

From Fig. 1 it can be seen that at $t=0$, cells which have reached $P_{2}$ in their cell-cycle are positioned at point $x=x_{2}$ on the axis. Without loss of generality, at time $t=0$ let $x_{2}$ be the origin. As an initial condition for $u$, we set $u=0$ for cells which have yet to reach $P_{2}$ (cells in regions I and II), while for cells in region III $(x<0)$ the stable steady state value of $u$ is chosen, that is

$$
u= \begin{cases}\tilde{u}_{2} & \text { if } x \leqslant 0 \\ 0 & \text { if } x>0\end{cases}
$$

where $\tilde{u}_{2}$ is given by (3.2).

The initial condition for $v$ is based on the distribution of $v$ just before the next signal is emitted. The process of signalling can be broken down into two stages for which approximate analytical solutions can be found. An approximate analytical solution for $v$ between signals is derived in Section 4 and is used as the initial condition for $v$ in the numerical solutions (Section 5).

\section{Analysis of the signalling equation}

The spatio-temporal dynamics of $v(x, t)$ is one of the key influences in the patterning process, and it is now shown that there are two possible cases depending on the 


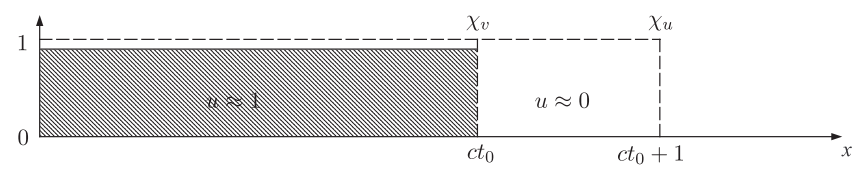

(a) The heaviside functions $\chi_{v}=H(c t-x)$ and $\chi_{u}=H(c t-x+1)$ at time $t_{0}$. The shaded region represents points where $u \approx 1$. At point $x=c t_{0}, u$ drops from a high value, $u \approx 1$, to a low value, $u \approx 0$.

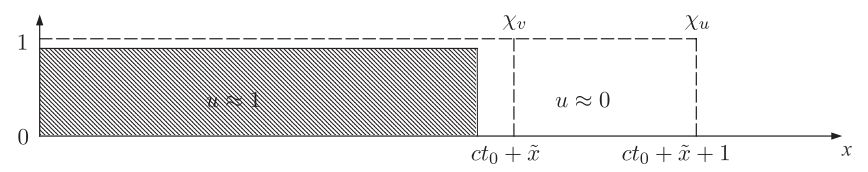

(b) The heaviside functions $\chi_{v}=H(c \tilde{t}-x)$ and $\chi_{u}=H(c \tilde{t}-x+1)$ at a slightly later time. Notice that the heaviside function $\chi_{v}$ has moved into the region where $u \approx 0$.

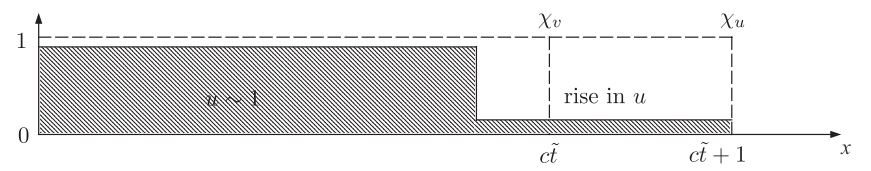

(c) The heaviside functions $\chi_{v}=H(c \tilde{t}-x)$ and $\chi_{u}=H(c \tilde{t}-x+1)$ at time $t=\tilde{t}$. The shaded region represents points where $u \sim 1$. At point $x=c \tilde{t}+1, u$ drops from a high value to a low value, $u \approx 0$.

FIG. 4. Schematic behaviour of the Heaviside functions along with changes in the value of $u$.

concentration of $u$. Analytical expressions are derived in each case and used in the analysis of the model equations. form:

Recall that the signalling equation is a reaction-diffusion equation of the following

$$
\frac{\partial v}{\partial t}=\frac{\chi_{v}(x, t)}{\epsilon+u}-v+D \frac{\partial^{2} v}{\partial x^{2}}
$$

where $\chi_{v}(x, t)=H(c t-x)$. For notational convenience we define the linear differential operator $L:=\frac{\partial}{\partial t}-D \frac{\partial^{2}}{\partial x^{2}}$. Then, (29) can be written as

$$
L v= \begin{cases}(\epsilon+u)^{-1}-v & \text { if } x \leqslant c t, \\ -v & \text { if } x>c t .\end{cases}
$$

The nonlinear source term was chosen to model negative feedback by $u$ on the production of $v$ : an increase in $u$ leads to a decrease in $v$, resulting in a reduction in $u$. This behaviour can be characterized schematically as in Fig. 4 which depicts the Heaviside functions along with changes in the value of $u$.

From Figs 4(a) and (b) we see that at time $t_{0}$, the Heaviside function $\chi_{v}$ begins to move into the region where $u \approx 0$. For $\epsilon \ll 1$ this leads to a rapid rise in $v$ at $x_{0}=c t_{0}$. We approximate the nonlinear source term in (30) by assuming that for cells posterior to 
$x_{0}=c t_{0}$, the level of somitic factor is close to its high stable steady state $\left(u \approx 1-\epsilon_{1}\right)$ while for cells anterior to $x=c t_{0}$, the level of somitic factor is approximately zero $\left(u \approx \epsilon_{2}\right)$, where $\epsilon_{1}, \epsilon_{2} \ll 1$. We approximate the equation for the signalling molecule by

$$
L v \approx \begin{cases}\left(1+\epsilon-\epsilon_{1}\right)^{-1} & x \leqslant c t_{0} \\ \left(\epsilon+\epsilon_{2}\right)^{-1} & c t_{0}<x \leqslant c t \\ 0 & x>c t .\end{cases}
$$

For $D \gg 1, v$ diffuses quickly, leading to a rise in $v$ for all points in the domain. At some notional time $t=\tilde{t}>t_{0}$ (see Fig. 4(b)) the increase in $v$ activates $u$ between $x=c t_{0}$ and $x=c \tilde{t}+1$. As $u$ begins to rise it inhibits $v$ production, and so the pulse in $v$ quickly falls. The above approximation (31) for (30) then breaks down. The next pulse occurs when $\chi_{v}$ passes point $x=c \tilde{t}+1$.

From Fig. 4(c) we see that in the region where the nonlinear source term is active $(x<c \tilde{t}), u \sim 1$. All points in this region have responded to a pulse of $v$. The parameter constraint $\epsilon \ll 1$, discussed previously, ensures that points which have already responded to a pulse do not trigger another pulse, only points where $u \approx 0$ should signal. Hence for the case when $u \sim 1$ throughout the region where $\chi_{v}$ is active, $u$ can be approximated by $1-\epsilon_{3}$ where $\epsilon_{3} \ll 1$. Equation (30) can then be approximated as

$$
L v \approx \begin{cases}\left(1+\epsilon-\epsilon_{3}\right)^{-1}-v & \text { if } x \leqslant c t \\ -v & \text { if } x>c t\end{cases}
$$

where $\epsilon_{3} \ll 1$. In the following section analytical expressions are derived to explain how both (31) and (32) behave in space.

\subsection{Spatial behaviour of a pulse in signalling molecule}

The spatial behaviour of $v$ in (31) is investigated by solving the ordinary differential equation

$$
D \frac{\mathrm{d}^{2} v}{\mathrm{~d} x^{2}}-v= \begin{cases}-\left(1+\epsilon-\epsilon_{1}\right)^{-1} & x \leqslant c t_{0} \\ -\left(\epsilon+\epsilon_{2}\right)^{-1} & c t_{0}<x \leqslant c t \\ 0 & x>c t\end{cases}
$$

subject to zero-flux boundary conditions at $x= \pm l$. For convenience we denote $c t_{0}=x_{0}$ and $c t=x_{0}+\tilde{x}$. Assuming the solution is continuously differentiable throughout the domain, (33) can be solved for $v(x)$ :

$$
\begin{aligned}
& v\left(x ; x_{0}, \tilde{x}\right)= \\
& \left\{\begin{array}{l}
(A-B) \sinh \left(\lambda\left(l-x_{0}\right)\right) \cosh (\lambda(l+x)) /(A B \sinh (2 \lambda l)) \\
-A \sinh \left(\lambda\left(l-x_{0}-\tilde{x}\right)\right) \cosh (\lambda(l+x)) /(A B \sinh (2 \lambda l))+1 / A \\
-A \sinh \left(\lambda\left(l-x_{0}-\tilde{x}\right)\right) \cosh (\lambda(l+x)) /(A B \sinh (2 \lambda l)) \\
-(A-B) \sinh \left(\lambda\left(l+x_{0}\right)\right) \cosh (\lambda(l-x)) /(A B \sinh (2 \lambda l))+1 / B \\
-(A-B) \sinh \left(\lambda\left(l+x_{0}\right)\right) \cosh (\lambda(l-x)) /(A B \sinh (2 \lambda l)) \\
-A \sinh \left(\lambda\left(l+x_{0}+\tilde{x}\right)\right) \cosh (\lambda(l-x)) /(A B \sinh (2 \lambda l))
\end{array} \quad x>x_{0}+\tilde{x}\right.
\end{aligned}
$$




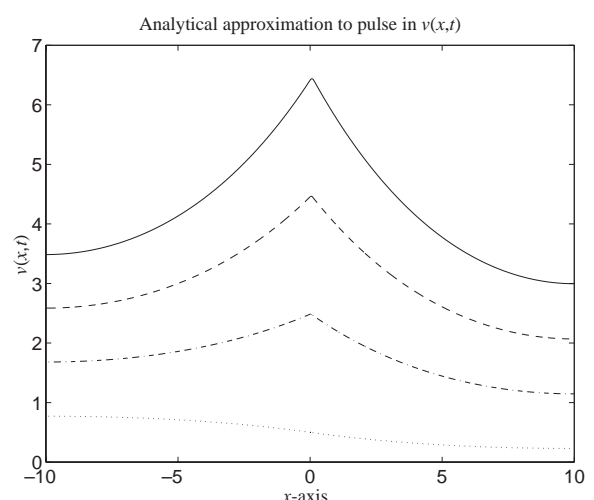

(a)

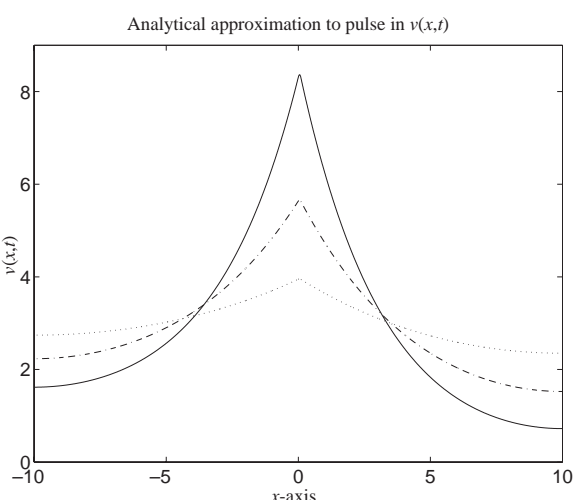

(b)

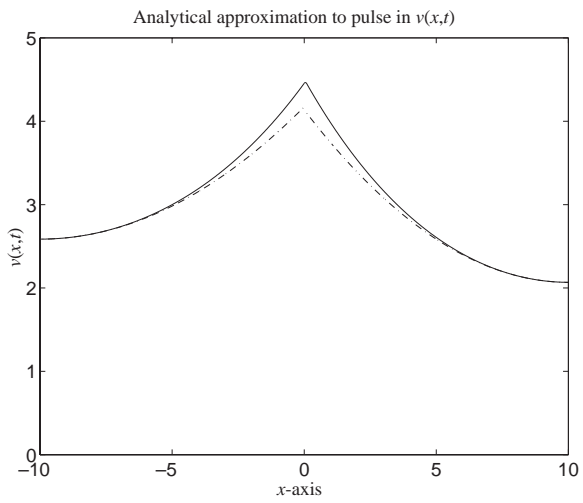

(c)

FIG. 5. Analytic solution (34) to the pulse $v(x, t)$ given by (33). (a) Varying $\tilde{x}$. Parameter values $\epsilon=\epsilon_{1}=\epsilon_{2}=$ $10^{-3}, D=50, x_{0}=0$. The dotted curve is the profile when $\tilde{x}=0 \cdot 0$, dash-dotted curve the profile for $\tilde{x}=0 \cdot 05$, dashed curve the profile for $\tilde{x}=0 \cdot 1$ and solid curve the profile for $\tilde{x}=0 \cdot 15$. (b) Varying diffusion. Parameter values $\epsilon=\epsilon_{1}=\epsilon_{2}=10^{-3}, x_{0}=0, \tilde{x}=0 \cdot 1$. The dotted curve is the profile for $D=100$, dash-dotted curve the profile for $D=25$ and solid curve the profile for $D=10$. (c) Cosh approximation. Parameter values $\epsilon=\epsilon_{1}=\epsilon_{2}=10^{-3}, x_{0}=0, \tilde{x}=0 \cdot 1$. The solid curve is the exact profile and the dash-dotted curve the cosh approximation using (35).

with $\lambda \equiv \sqrt{1 / D}, A=1+\epsilon-\epsilon_{1}$ and $B=\epsilon+\epsilon_{2}$. The expression (34) is plotted for various values of $\tilde{x}$ in Fig. 5(a).

Notice that the solution is not symmetrical about the origin. Although small in comparison with $1 /\left(\epsilon+\epsilon_{2}\right)$, the non-zero source term for $x<c t_{0}$ ensures that the signalling level is slightly higher posterior to the centre of the pulse. As $\tilde{x}$ increases the signalling profile changes as the width of the pulse increases. Recall that one of the key requirements in the model equations is that diffusion is large. In Fig. 5(a) we see that by increasing the diffusion coefficient $D$, the signal spreads across the domain.

On setting $D \gg 1, \lambda$ is small and the cosh function may be approximated by a quadratic polynomial,

$$
\cosh \{\lambda(l+x)\} \approx 1+\frac{1}{2}\{\lambda(l+x)\}^{2} .
$$


This approximation is depicted by the dash-dotted curve in Fig. 5(c), providing a reasonably good qualitative approximation to the full analytical solution (34).

\subsection{Spatial behaviour between pulses}

The spatial behaviour of $v$ between pulses is determined from (32) by solving the ordinary differential equation

$$
D \frac{\mathrm{d}^{2} v}{\mathrm{~d} x^{2}}-v \approx-\frac{H\left(x_{0}-x\right)}{1+\epsilon-\epsilon_{3}},
$$

where $x_{0}=c t$ and the discontinuity in the Heaviside function occurs at $x_{0}$. The Heaviside function leads to a discontinuity in the second derivative, that is

$$
\left.D \frac{\mathrm{d}^{2} v}{\mathrm{~d} x^{2}}\right|_{x_{0}+\varepsilon} ^{x_{0}-\varepsilon}-\left.v\right|_{x_{0}+\varepsilon} ^{x_{0}-\varepsilon}=\frac{1}{1+\epsilon-\epsilon_{3}} .
$$

Letting $\varepsilon \rightarrow 0$, for $v$ continuous, we have the following jump condition across $x=x_{0}$ :

$$
\left[\frac{\mathrm{d}^{2} v}{\mathrm{~d} x^{2}}\right]=\left.\frac{\mathrm{d}^{2} v}{\mathrm{~d} x^{2}}\right|_{x_{0+}}-\left.\frac{\mathrm{d}^{2} v}{\mathrm{~d} x^{2}}\right|_{x_{0-}}=-\frac{1}{D\left(1+\epsilon-\epsilon_{3}\right)} .
$$

Using continuity across $x_{0}$ along with the jump in the second derivative, (36) can be solved analytically, giving

$$
v\left(x, x_{0}\right)=\left\{\begin{array}{cc}
\left(A-\frac{1}{1+\epsilon-\epsilon_{3}}\right) \frac{\cosh (\lambda(l+x))}{\cosh \left(\lambda\left(l+x_{0}\right)\right)}+\frac{1}{1+\epsilon-\epsilon_{3}}, & -l<x<x_{0}, \\
A \frac{\cosh (\lambda(l-x))}{\cosh \left(\lambda\left(l-x_{0}\right)\right)}, & x_{0}<x<l,
\end{array}\right.
$$

with constants $A$ and $\lambda$ given by

$$
A \equiv \frac{\left(\frac{1}{1+\epsilon-\epsilon_{3}}\right) \tanh \left(\lambda\left(l+x_{0}\right)\right)}{\tanh \left(\lambda\left(l-x_{0}\right)\right)+\tanh \left(\lambda\left(l+x_{0}\right)\right)}, \quad \lambda \equiv \sqrt{\frac{1}{D}} .
$$

On setting $x_{0}=0$, the solution simplifies to

$$
v(x, 0)=\frac{H(-x)}{1+\epsilon-\epsilon_{3}}+A \cosh (\lambda(l-|x|)),
$$

where

$$
A \equiv \frac{\operatorname{sign}(x)}{2\left(1+\epsilon-\epsilon_{3}\right) \cosh (\lambda l)}, \quad \lambda \equiv \sqrt{\frac{1}{D}} .
$$

Solution (39) is plotted for various values of $x_{0}$ in Fig. 6(a). As $x_{0}$ increases the profile changes, in particular we see that $v(x) \simeq 1$ when $x_{0}=10$.

We would expect the solution of (39) to agree qualitatively with spatial plots of (30) before and after a pulse in $v(x, t)$. The solutions for various values of $D$ and $x_{0}$ are plotted in Fig. 6(b). For $D$ small there is a smooth transition from zero to one, across $x_{0}$. On choosing $D$ large we see that $v \sim 0.5$. 


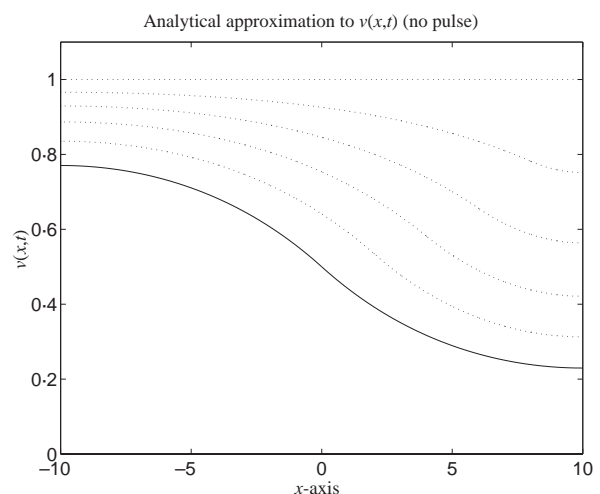

(a)

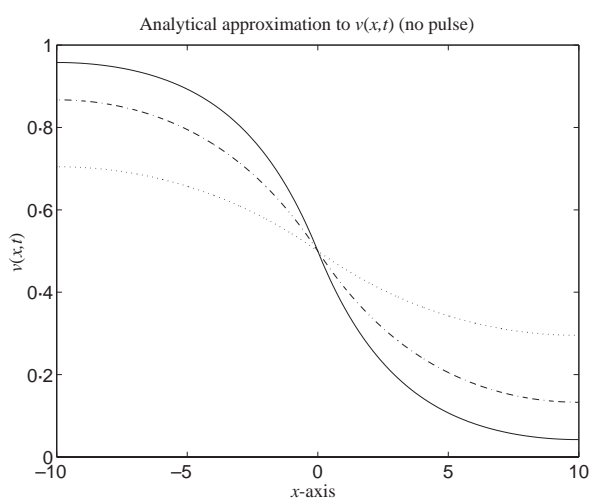

(b)

FIG. 6. Analytical solution (39) of (36). (a) Varying $x_{0}$. Parameters $D=50, \epsilon=10^{-3}, \epsilon_{3}=10^{-3}$. The solid curve is the profile for $x_{0}=0$ and the dotted curves are profiles for $x_{0} \equiv c t=2,4,6,8,10$. (b) Varying diffusion. Parameter $x_{0}=0$. The solid curve is the profile for $D=10$, dash-dotted the profile for $D=25$ and dotted curve the profile for $D=100$.

Analytical expression for the rise in $v$. We now seek an analytical solution to (31). Thereby, we consider

$$
\frac{\partial v}{\partial t}-D \frac{\partial^{2} v}{\partial x^{2}}-v \approx \begin{cases}\left(1+\epsilon-\epsilon_{1}\right)^{-1} & x \leqslant c t_{0} \\ \left(\epsilon+\epsilon_{2}\right)^{-1} & c t_{0}<x \leqslant c t \\ 0 & x>c t\end{cases}
$$

for $t \geqslant t_{0}$. Without loss of generality, we take $t_{0}=0$.

Make a change of variables, so that $y(x, t)=v(x, t) \exp (t / \eta)$ and $y(x, t)$ satisfies

$$
\frac{\partial y}{\partial t}-D \frac{\partial^{2} y}{\partial x^{2}} \approx \begin{cases}\mathrm{e}^{t}\left(1+\epsilon-\epsilon_{1}\right)^{-1} & x \leqslant 0 \\ \mathrm{e}^{t}\left(\epsilon+\epsilon_{2}\right)^{-1} & 0<x \leqslant c t \\ 0 & x>c t\end{cases}
$$

with the same initial and boundary conditions as $v(x, t)$. Using Green's functions to solve the above we have

$$
\begin{aligned}
v(x, t)= & \frac{1}{2\left(1+\epsilon-\epsilon_{1}\right)} \int_{0}^{t}\left\{1-\operatorname{erf}\left(\frac{x}{\sqrt{4 D\left(t-t^{\prime}\right)}}\right)\right\} \mathrm{e}^{-\left(t-t^{\prime}\right)} \mathrm{d} t^{\prime} \\
& +\frac{1}{2\left(\epsilon_{1}+\epsilon_{2}\right)} \int_{0}^{t}\left\{\operatorname{erf}\left(\frac{x}{\sqrt{4 D\left(t-t^{\prime}\right)}}\right)-\operatorname{erf}\left(\frac{x-c t^{\prime}}{\sqrt{4 D\left(t-t^{\prime}\right)}}\right)\right\} \mathrm{e}^{-\left(t-t^{\prime}\right)} \mathrm{d} t^{\prime} \\
& +\int_{-\infty}^{\infty} \frac{\mathrm{e}^{-t}}{\sqrt{\pi}} v_{0}(x-\sqrt{4 D t} u) \mathrm{e}^{-u^{2}} \mathrm{~d} u
\end{aligned}
$$

where $v_{0}(x)$ gives the initial conditions for $v$. 


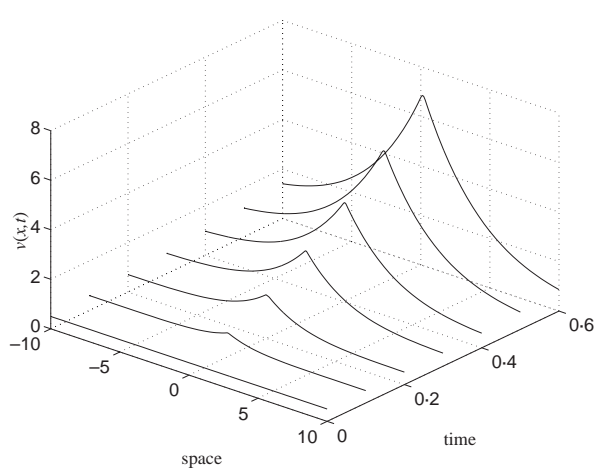

(a)

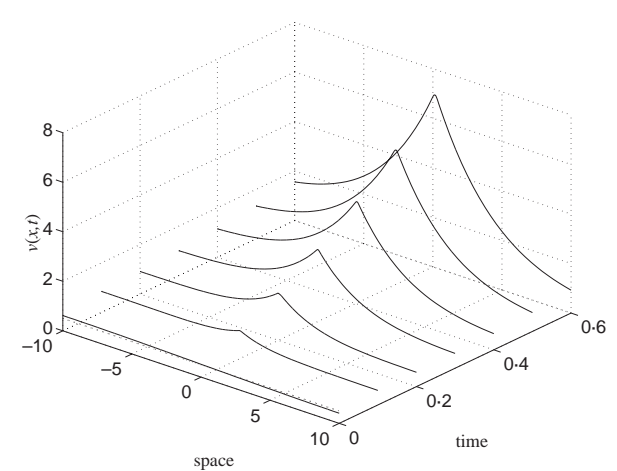

(b)

FIG. 7. Analytical solution (45) with parameter values $\epsilon=\epsilon_{1}=\epsilon_{2}=10^{-3}, D=50, c=0 \cdot 5$. The solution is plotted at time intervals of $\Delta t=0 \cdot 1$. (a) Analytical solution of $v(x, t)$. The initial condition is $v(x, 0)=0 \cdot 5$ (b) Initial behaviour of $v(x, t)$. The initial condition is given by solution (39). The dashed line represents the initial condition, $v(x, 0)=0 \cdot 5$, shown for comparison.

\section{Numerical solutions}

The model equations pose two main difficulties for numerical simulations: (1) the Heaviside function present in the equation for $u$ introduces a sharp discontinuity in the solution, and (2) the system is of mixed type: the $u$ equation is hyperbolic, while the $v$ equation is parabolic. A sophisticated numerical scheme was developed to solve the model equations in one spatial dimension based on the finite element method (details to be reported elsewhere).

The computational domain $\Omega$ is chosen to be $[-10,10]$. In the following numerical simulations we subdivide $\Omega$ into 500 uniform intervals, $h=0.04$, and choose a time-step $k=0 \cdot 1$. The mesh parameters $h$ and $k$ have been chosen by repeated numerical simulation to ensure that the analytical solution of our model system is accurately represented by its finite element approximation, without the need for excessive computation. Indeed, our numerical experiments indicate that a time-step of less than or equal to 0.1 is needed to accurately simulate the pulse-like signal in $v$, with the spatial mesh chosen fine enough to adequately resolve the localized jumps in $u$.

\subsection{Initial conditions used in numerical simulations}

The initial conditions for $u$ and $v$ were discussed in Section 3.6. The initial condition for $u$ is given by (28). For points $x<0$ we set $u=0$, while for $x>0$ we choose the stable steady state value of $u$,

$$
\tilde{u}_{2}=\frac{1}{2}\left[1+\sqrt{1-\frac{4 \gamma}{\kappa}}\right] \text {, }
$$




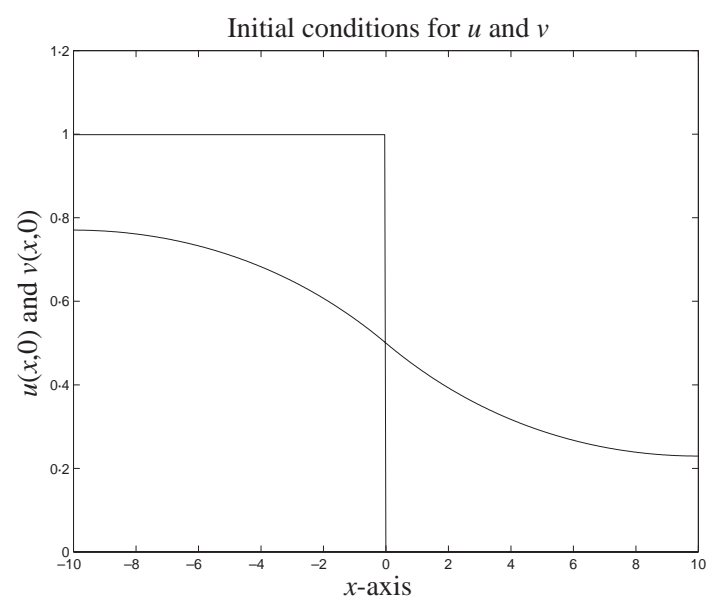

FIG. 8. Initial conditions $u(x, 0)$ and $v(x, 0)$.

which is approximately 1 for parameters $\kappa=10$ and $\gamma=10^{-2}$. Thus our initial condition for $u$ is simply

$$
u(x, 0)= \begin{cases}1 & \text { if } x \leqslant 0 \\ 0 & \text { if } x>0 .\end{cases}
$$

A suitable choice of initial condition for $v(x, t)$ is its distribution just before the next signal is emitted. A good estimate of this is given by (39) with $x_{0}=0 \cdot 0, l=10$ and $D=50$,

$$
v(x, 0)=H(-x)+A \cosh (\lambda(10-|x|)),
$$

where the constants $A$ and $\lambda$ are given by

$$
A \equiv \frac{\operatorname{sign}(x)}{2 \cosh (10 \lambda)}, \quad \lambda \equiv \sqrt{\frac{1}{50}} .
$$

The parabolic equation (5b) is subject to zero flux boundary conditions

$$
\frac{\partial v}{\partial x}=0, \quad x=x_{0}, x_{N}, t \in[0, T],
$$

where $T$ denotes the time at which we stop the simulation. The initial conditions for $u$ and $v$, as described by (47) and (48), respectively, are shown in Fig. 8.

A typical numerical solution to (5a) and (5b) with zero-flux boundary condition and initial conditions (48) and (47) is shown in Fig. 9. We see that a pulse in $v$ is emitted at $x \approx 0$ leading to a jump in $u$ between points $x \approx 0$ and $x \approx 1$. This jump in $u$ corresponds to a collection of cells that will later form a somite. The first pulse at $x \approx 0$ is followed by a second at $x \approx 1$ and a third at $x \approx 2$, causing a series of jumps in $u$ along the $x$-axis. 


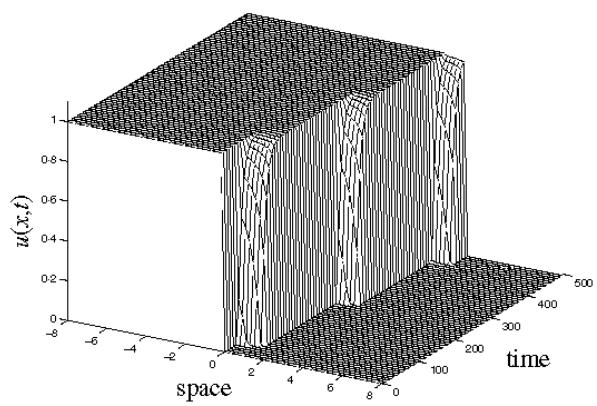

(a) Jumps in $u(x, t)$

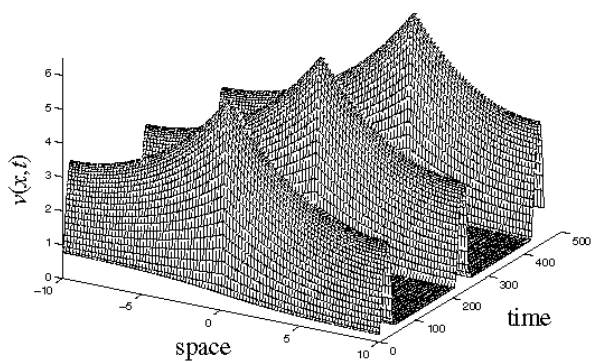

(b) Pulses in $v(x, t)$

FIG. 9. Numerical solution of (5a) and (5b) for $0 \leqslant t \leqslant 500$. Parameter values are $\mu=10^{-4}, \gamma=10^{-2}, \kappa=$ $10, c=5 \times 10^{-3}, \epsilon=10^{-3}, D=50$.

The process begins with a peak in $v$ at the position of $P_{2}$, quickly followed by a surge of $v$ throughout the spatial domain. The level of $v$ then decreases rapidly to a constant level. In response to this pulse-like signal, a fairly rapid increase in $u$ occurs between $P_{1}$ and $P_{2}$. Thus we have a wavefront of the somitic factor $(u)$ moving down the axis in jumps, as successive groups of cells are triggered. Note that the level of $u$ rises at approximately the same time in all cells between $P_{1}$ and $P_{2}$ when a signal is emitted. According to the model, this results in the coordinated segmentation of a somite.

\subsection{Comparison between the analytical and numerical results}

In Section 4 the signalling process was broken down into two stages. The dynamics of the pulse-like signal and the distribution of the signalling molecule between pulses were investigated. We now compare the approximate analytical results derived there with the numerical solution of the full model equations (5a) and (5b). The rise of the first pulse in $v(x, t)$ is depicted in Fig. 10(a). The analytical approximation to the pulse (34), derived in Section 4, closely matches the initial rise in $v(x, t)$ (see Figs 5 and 7). However, on closely examining Fig. 7, we see that although there is good qualitative agreement, the rise in $v$ is considerably faster than that observed numerically. Even for a choice of $\epsilon=0.02$ (which is an order of magnitude greater than that chosen in Fig. 10(a)) the $\delta$-function approximation we use to capture the pulse-like behaviour exaggerates the rise in $v$. Although useful, the approximation cannot account for the fact that an increase in $u$ inhibits $v$. This effect is depicted in Fig. 12 where the rise in $v$ slows as $u$ increases (Fig. 13(a)).

In Fig. 10(b) each profile represents the solution either before or after a pulse in $v(x, t)$. The solution is plotted over a considerably longer time scale than in Fig. 10(a). As the Heaviside function $\chi_{v}$ moves across the domain the profile in $v(x, t)$ tends to 1 . The analytical expression for the spatial behaviour of the signalling molecule between pulses (34) matches the behaviour observed (Fig. 6). 


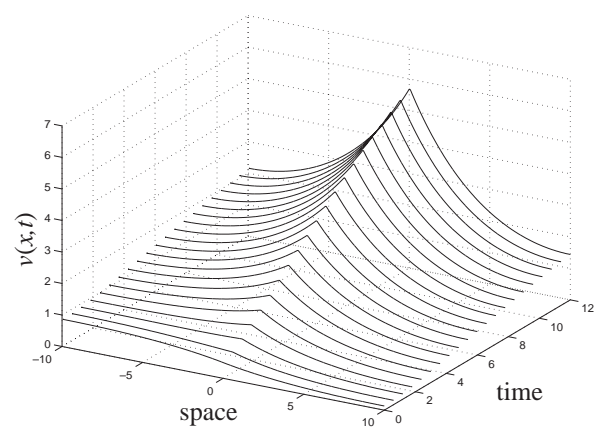

(a)

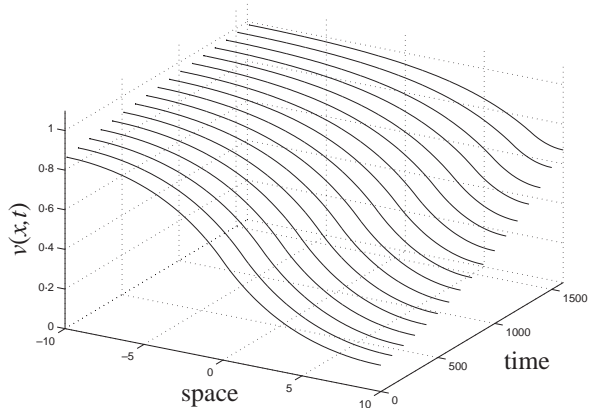

(b)

FIG. 10. Numerical solution of (30) on $[-10,10]$ subject to zero flux boundary conditions, with parameter values as in Fig. 9. The spatial profile is plotted at selected time intervals. Note that in (a) and (b) the solution is plotted over different timescales. The number of discretization points is 401 and the time-step is $0 \cdot 1$ in each case. (a) The first peak in $v(x, t)$ for $0 \leqslant t \leqslant 12$. Points where $u \approx 0$ give rise to a pulse in $v$ centred at $x=0$. (b) $v(x, t)$ for $0 \leqslant t \leqslant 1600$. Points where $u \approx 1$ lead to a distribution of $v \sim 1$ (the distribution of $v(x, t)$ either before or after a pulse).

\section{Discussion}

In this paper we have summarized the derivation of the mathematical model of Collier et al. (2000) for somitogenesis based on the cell-cycle model of Stern et al. (1988). We have presented an analysis and numerical simulations of the model equations, which (i) enable us to place constraints on model parameters, (ii) provide a greater understanding of the signalling process, and (iii) allow us to clearly understand which parameters influence somite length. We now present these insights in more detail.

\subsection{Model requirements and parameter constraints}

One important point to make is that parameter bounds were derived to ensure that the cells of a potential somite adhere at about the same time, resulting in the coordinated segmentation of a discrete somite. The parameter constraints derived in Section 3 can be summarized as follows:

$$
\begin{array}{ll}
\text { I } & \kappa \gg 1 \text { and } \gamma<\frac{\kappa}{4} \\
\text { II } & 1<\theta_{l}<\frac{1}{\epsilon+\frac{\gamma}{4 \kappa}} \\
\text { III } & \theta_{l} \ll \frac{1}{\epsilon} \\
\text { IV } & \epsilon \ll \tilde{u}_{2} \\
\text { V } & D \gg 1 .
\end{array}
$$

The parameters chosen in Fig. 9 satisfy each of these constraints. 
On choosing $\kappa=10 \gg 1$ we obtain a pulse-like signal. For $\gamma=10^{-2}$, the requirement that $\gamma<\kappa / 4$ is easily satisfied. By imposing this constraint we ensure that $u$ changes from a low to a high level when $v$ reaches the threshold level (as shown in Fig. 13).

If any of the parameter constraints are violated the model fails to generate the required spatio-temporal pattern of somites. For example, our lower bound for the threshold level of signalling molecule, $\theta_{l}$, is given by (52). This is sufficiently high to ensure that $u$ production is not activated for $v \approx 1$. In Fig. 11(a)-(b) we choose $\mu=10^{-5}$ and $\gamma=0 \cdot 2$; with these changes the lower bound for the threshold level, $\theta_{l}=5 \times 10^{2}$. Furthermore,

$$
\theta_{l}<\frac{1}{\epsilon+\frac{\gamma}{4 \kappa}} \approx 2 \times 10^{2}
$$

violating condition (II). As a result even a very high level of $v$ fails to activate $u$ production.

For the parameters in Fig. 9 condition (III) is easily satisfied,

$$
\theta_{l}=2.5 \ll \frac{1}{\epsilon}=10^{3} .
$$

It is important that $\theta_{l}$ should be significantly smaller than $1 / \epsilon$. For example, on choosing $\gamma=0.05$ (or $\epsilon=5 \times 10^{-3}$ ), the signalling process fails (see Fig. 11(c)-(d)). Though $v$ rises rapidly it fails to activate $u$ in the manner required by the model, instead we observe a partial jump in $u$. Given $\epsilon \ll 1, v$ becomes large only for $u \approx 0$, points along the axis where $u \approx 1$ (those that have responded to a previous pulse of $v$ ) do not cause the next pulse to occur, thus satisfying the requirement that cells which have already responded to a signal and hence go on to form a somite must not respond again, only cells in which $u$ is low can respond to a signal.

In Fig. 9 a value of $D=50$ ensures that at time $\tilde{t}=8$ the size of a somite is determined by points $x=0.0$ and $x=1+c \tilde{t}=1.04$ rather than by the extent to which the signal spreads. Biologically this corresponds to the requirement that the signal must spread far enough to activate $u$ production in all cells between $P_{1}$ and $P_{2}$. In Fig. 11(e)-(f) we choose $D=5$ (violating the requirement that diffusion is high). The pulse in $v$ is slow to rise, leading to a delay in specification. More importantly, for points to the left of $x \approx 1$, the level of $v$ is never high enough to activate $u$ in sufficient quantities. The length of somite specified is shorter than 1 .

\subsection{Explanation of the signalling process}

The signalling process is explained by considering cross-sections (Fig. 12) of the numerical solution in Fig. 9. Initially $u=0$ for all points $x \geqslant 0$ and $v \sim 1$ throughout the domain (Fig. 8). Our choice of $\epsilon=10^{-3}$ ensures that as the Heaviside function $\chi_{v}=H(x-c t)$ moves to the right at speed $c=0 \cdot 005$, there is a rapid rise in $v$ in points where $u=0$. This leads to a pulse in $v$ centred at $x \approx 0$ as shown in Figs 9(b) and 12(a).

With the parameters in Fig. 9 we see that $\theta_{l}$, our lower bound for $\theta$, the threshold that must be exceeded by $v$ to activate $u$ production, is given by

$$
\theta_{l}=\frac{\gamma}{4 \mu \kappa}=2 \cdot 5 .
$$




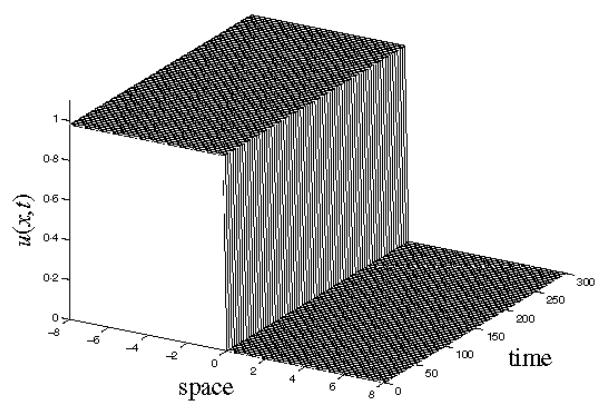

(a) No change in $u(x, t)$

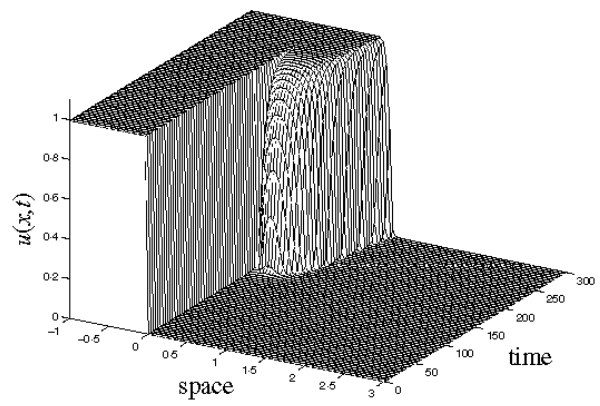

(c) Jump in $u(x, t)$

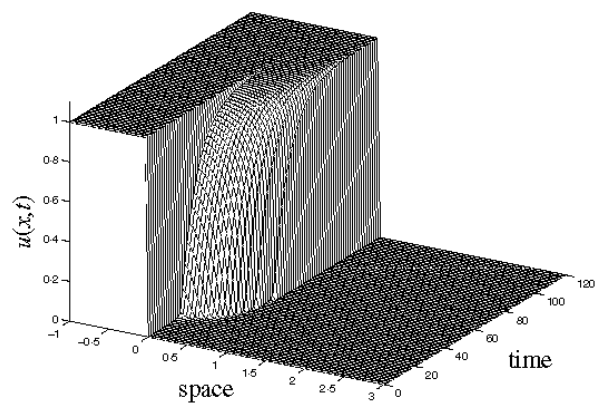

(e) Jump in $u(x, t)$

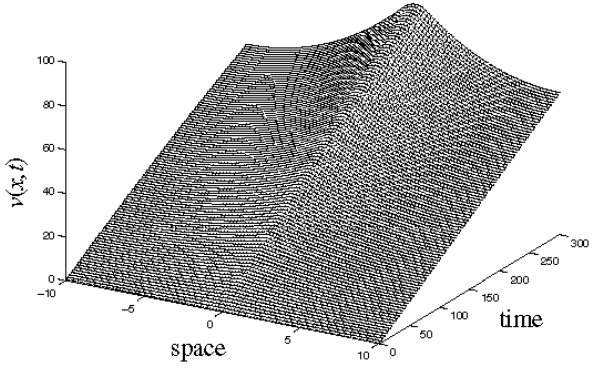

(b) Rise in $v(x, t)$

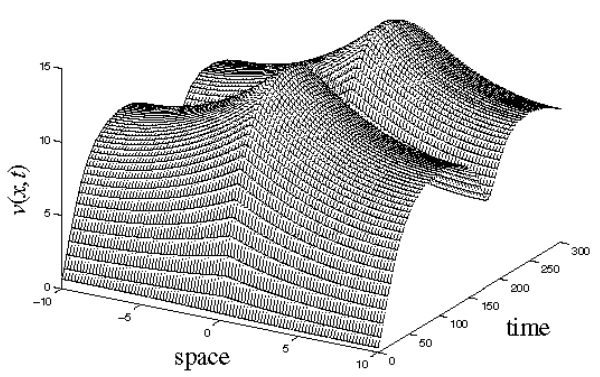

(d) Rise in $v(x, t)$

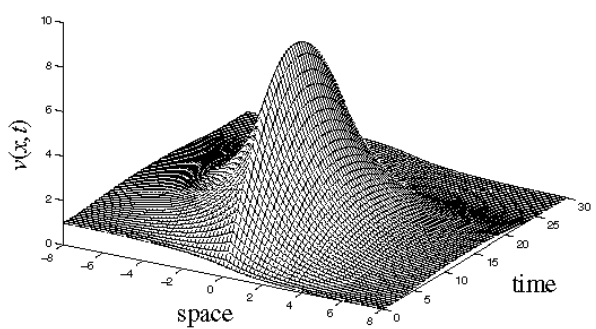

(f) Pulse in $v(x, t)$

FIG. 11. Numerical solution of (5a) and (5b) for $0 \leqslant t \leqslant 300$ : (a)-(b) Parameter values $\mu=10^{-5}, \gamma=$ $0 \cdot 2, \kappa=10, c=5 \times 10^{-3}, \epsilon=10^{-3}, D=100$ (violating condition II). For $\mu=10^{-5}$ and $\gamma=0.2$ a high level of $v$ fails to activate $u$ production. (c)-(d) Parameter values $\mu=10^{-4}, \gamma=5 \times 10^{-2}, \kappa=10, c=$ $5 \times 10^{-3}, \epsilon=10^{-3}, D=100$ (violating condition III). For $\gamma=0.05$ the level of $v$ is not high enough to activate $u$ production at all points. (e)-(f) Parameter values $\mu=10^{-4}, \gamma=10^{-2}, \kappa=10, c=5 \times 10^{-3}, \epsilon=$ $10^{-3}, D=5$ (violating requirement $\mathrm{V}$ ). Diffusion of $v$ is insufficient to activate $u$ at all points.

At some time $t=\tilde{t} \approx 10$ (Fig. 12(a)), before it reaches its peak, $v$ rises above the threshold leading to a rapid increase in $u$ production at all points between $x=0$ and $x=1+c \tilde{t} \approx$ 1.04. As $u$ increases the rise in $v$ slows; at $t \approx 25, u$ is $\mathrm{O}(\epsilon)$ (Fig. 13(a)), $v$ has reached its 

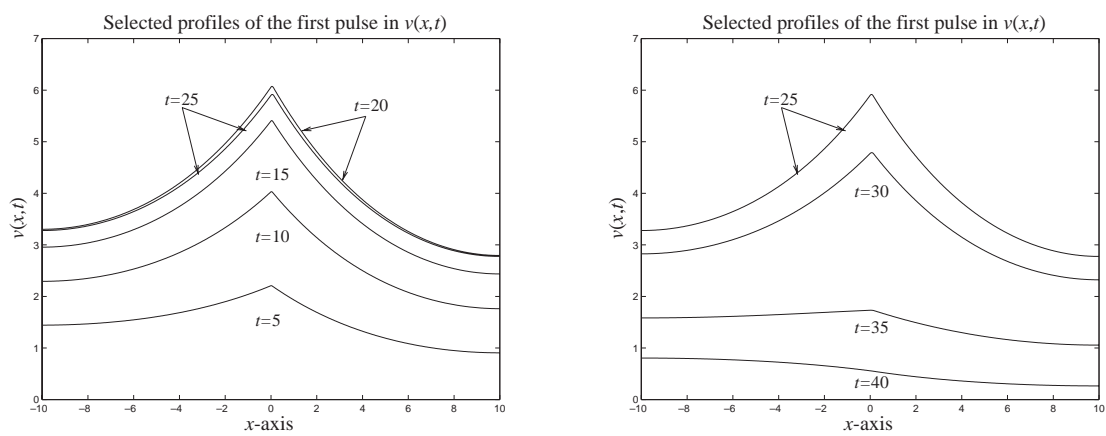

FIG. 12. Finite element solution for $v(x, t)$ at selected time intervals. Parameter values as in Fig. 9. (a) The rise in $v$ for $5 \leqslant t \leqslant 25$. (b) The fall in $v$ for $25 \leqslant t \leqslant 40$.

peak (Fig. 12(b)). In Fig. 13(b) we see that $u$ begins to rise rapidly as points between $x=0$ and $x=1+c \tilde{t}$ make the transition towards the stable steady state $\tilde{u}_{2} \approx 1$. This rapid rise inhibits $v$ production, and $v$ quickly falls, returning to $v \sim 1$. At $t=40, u$ is $\mathrm{O}\left(10^{-1}\right)$ for all points between $x=0$ and $x=1+c \tilde{t}$ and $v$ is $\mathrm{O}(1)$.

From Fig. 13(a)-(b) it is clear that although $u$ becomes $\mathrm{O}(1)$ very quickly in response to a pulse in $v$, after $t=70, u$ rises slowly (see Fig. 13(c)). Activation of $u$ by $v$ and saturation for large $u$ is consistent with our model requirements. It is not until time $t \approx 80$ that the region $0<x<1+c \tilde{t}$ is specified as somitic.

At time $t=208$, the Heaviside function $\chi_{v}$ reaches point $x=1.04$. Low values of $u$ begin to activate production of $v$ leading to a second pulse in $v$. The process described above is repeated and a second jump in $u$ follows.

\subsection{Somite length}

There are delays between signalling, activation of $u$ and specification. At time $t=0$, points at $x=0$ correspond to cells which have reached point $P_{2}$ in their cell-cycle, while points at $x=1$ correspond to cells which have reached $P_{1}$ (see Fig. 1). The model states that a pulse of $v$ at $x=0$ activates production of $u$, leading to a region of specification between points $x=0$ and $x=1$. From the description above we saw that although production of $v$ begins almost immediately, what determines somite length in our mathematical formulation is the time at which the pulse in signalling molecule rises above the threshold required to activate $u$. In Fig. 13(a) we see that non-trivial production of $u$ begins at $t \approx 10$. This confirms numerically the point made in the previous section, namely that in the model, cells gain the potential to form a somite a short time after the signal is emitted. Figures 9(a) and 13(d) show the region of specification corresponding to three distinct somites. In Fig. 13(d) the length of each somite is approximately 1.04.

In the cell-cycle model, cells are assumed to be roughly in age (cell-cycle) synchrony and the size of a somite depends on the distance between the time points $P_{1}$ and $P_{2}$ within the cycle. Recently, Dubrulle et al. (2001) have suggested that an interaction between FGF8 signalling and the segmentation clock controls somite size. If FGF-8 is applied to the PSM, the FGF-8 gradient extends caudally further than normal, preventing more PSM cells 

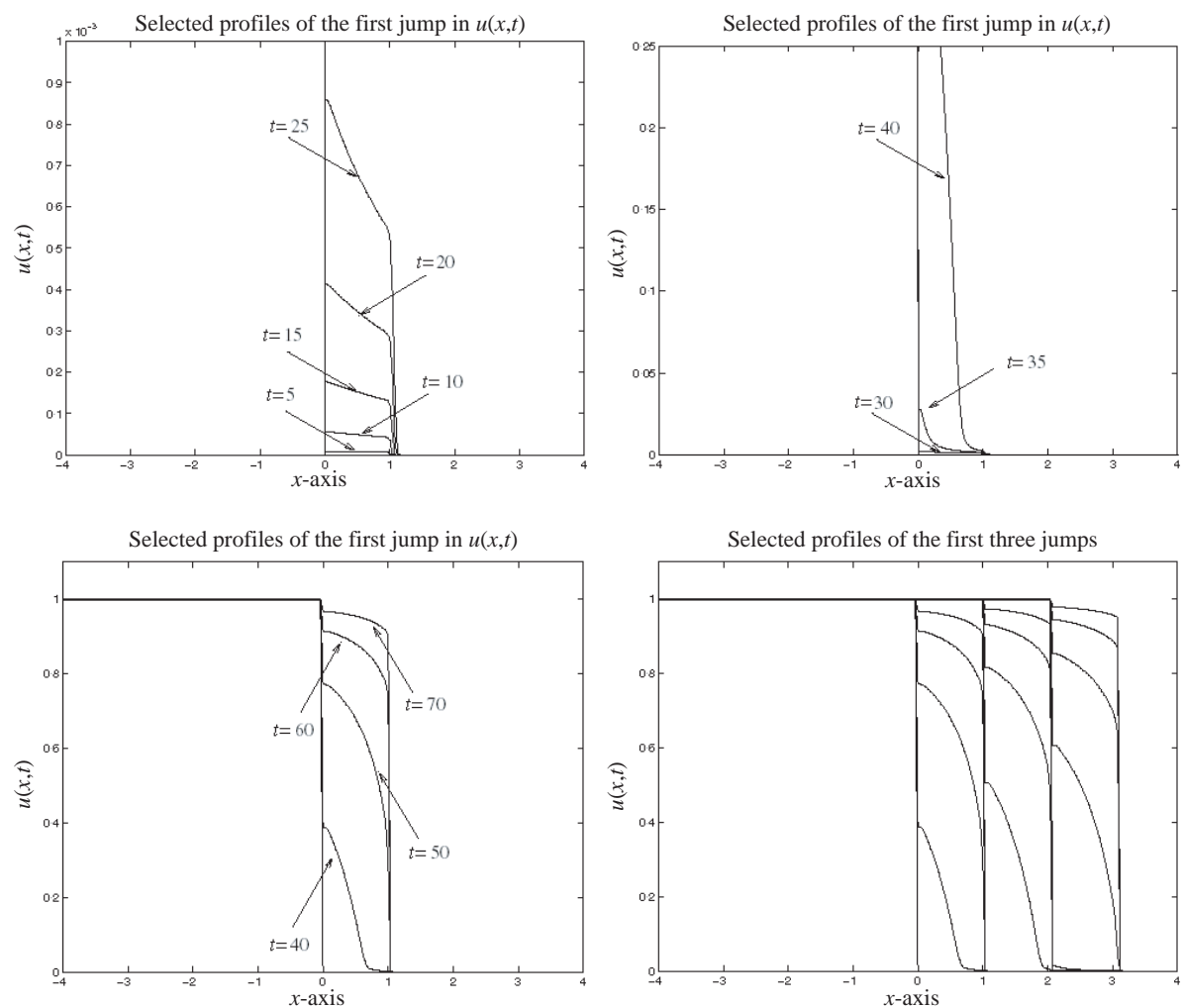

FIG. 13. Finite element solution for $u(x, t)$ at selected time intervals. Parameter values as in Fig. 9. (a) Slow initial rise in $u$ for $5 \leqslant t \leqslant 25$. (b) Subsequent rapid rise in $u$ for $25 \leqslant t \leqslant 40$. (c) First jump in $u$ for $0 \leqslant t \leqslant 70$. Although initially fast, by time $t=70$ the rise in $u$ begins to slow. (d) First three jumps in $u$ for $0 \leqslant t \leqslant 490$. By time $t=490$ a third somite has been specified. Each somite specified has an approximate length of 1.04.

from contributing to a somite. Baker et al. (2003) have developed a new version of the cellcycle model to incorporate the interaction between FGF-8 signalling and the segmentation clock. We are presently testing this hypothesis by extending the mathematical formulation of the cell-cycle model, along with its analysis and numerical simulations.

\subsection{Challenging analytical problems}

The greatest remaining challenge with this mathematical formulation of the cell-cycle model for somitogenesis is the undertaking of a rigorous asymptotic analysis-something that has thus far not been addressed. There are a number of reasons for this.

The model equations are highly nonlinear and the dynamics of each dependent variable is highly dependent on the dynamics of the other. It has so far proved impossible to separate either variable from the other in a manner which would allow further productive analysis. Although there is a threshold level for the somitic factor, $u$, above which it will grow with very little dependence on signalling levels, the crux of our model is about understanding 
which cells experience signalling levels sufficient to allow them to reach this threshold and also the manner in which they do this.

Another problem to be overcome is isolating the multiple timescales involved; it is anticipated that there are at least three independent timescales that exist. The relationships between the model parameters needs to be explored and this is something that is difficult to justify biologically. To date, we have been unable to find a reasonable matching between the different regions of the model, although we are currently in the process of finding approximations for the somitic factor. These approximations cover much of the process of somitic factor production and we hope they will give us greater insight into the signalling process; perhaps allowing us to produce some more rigorous analysis in the future.

\section{Conclusions}

We have formulated our model in one spatial dimension to allow a detailed investigation of the model behaviour. The results are in agreement with a number of biological observations (see Schnell et al., 2000; Collier et al., 2000; Schnell et al., 2002, for further details). The analysis indicated that the cell-cycle mechanism can indeed give rise to the periodic pattern of somites observed in normal embryos. Furthermore, the model allows us to understand in more detail the signalling process and to determine which parameters control somite size. However, on using a one-dimensional domain, we cannot account for the fact that only some cells in the embryo are destined to form somites (and hence be capable of producing and responding to the signalling cues in the cell-cycle model). Moreover, this model does not account for cell motion. In one dimension we implicitly assume that all cells along the $x$-axis eventually form part of a somite. Contrary to prevailing views, recent experimental evidence shows that cell movement plays an important role in somite formation (Kulesa $\&$ Fraser, 2002). By extending the model to two dimensions and including cell movement we can investigate different modes of cell movement and can allow for the possibility that some cells are not incorporated into somites (details to be presented elsewhere).

It is important to emphasize that we are not proposing the cell-cycle model as the ultimate explanation for somite formation. Baker et al. (2003) have recently developed an extension of the cell-cycle model which could explain the effects of FGF-8 during somitogenesis. However, they have also shown that, with suitable modification, other models such as Cooke and Zeeman's clock and wavefront model (Cooke \& Zeeman, 1976) and Meinhardt's reaction-diffusion model (Meinhardt, 1982, 1986) can be viewed as consistent with the observations and so it is, we feel, a mistake to consider only one model, which seems presently to be the case. Our mathematical model represents a general signalling process in which cells become somitic after being triggered within the timewindow of the segmentation clock. This mathematical formulation and our analysis can be extended to study other somite formation models.

\section{Acknowledgements}

We thank two anonymous referees for helpful comments. We are also grateful to Dr D. J. Gavaghan (Computing Laboratory, University of Oxford) and Dr P. Houston (Department of Mathematics and Computer Science, University of Leicester) for their help with the numerical solutions. DMI was funded by a Marie Curie Fellowship as part of the 
Training and Mobility of Researchers (TMR) programme administered by the European Commission. SS acknowledges support from the José Gregorio Hernández Award (Academia Nacional de Medicina, Venezuela; Pembroke College, Oxford), ORS Award (Committee of Vice-Chancellors and Principals of the Universities the United Kingdom), Programa de Cofinanciamiento Institucional IVIC-CONICIT (CONICIT, Venezuela), Lord Miles Senior Scholarship in Science (Pembroke College) and the Research Training Fellowship Programme in Mathematical Biology (Grant No. 069155) of the Wellcome Trust (London). REB would like to thank EPSRC for a studentship in Mathematics, and Wadham College (Oxford) for a Wadham College Senior Scholarship. PKM was supported by a Royal Society/Leverhulme Trust Senior Research Fellowship for part of this work.

Part of this document has been typed with the aid of peditPro provided by the courtesy of PaulComputing (http://www.paulcomputing.com).

\section{REFERENCES}

BAKER, R. E., SCHNELL, S. \& MAINI, P. K. (2003) Formation of vertebral precursors: past models and future predictions. J. Theor. Med., 5, 23-35.

Collier, J. R., Mcinerney, D., Schnell, S., Maini, P. K., Gavaghan, D. J., Houston, P. \& STERN, C. D. (2000) A cell cycle model for somitogenesis: mathematical formulation and numerical simulations. J. Theor. Biol., 207, 305-316.

COOKE, J. (1978) Somite abnormalities caused by short heat shocks to pre-neurula stages of xenopus laevis. J. Embryol. Exp. Morphol., 45, 283-294.

CoOKe, J. \& ZEEMAN, E. C. (1976) A clock and wavefront model for control of the number of repeated structures during animal morphogenesis. J. Theor. Biol., 58, 455-476.

Dubrulle, J., MCGRew, M. J. \& PourquiÉ, O. (2001) FGF signaling controls somite boundary position and regulates segmentation clock control of spatiotemporal hox gene activation. Cell, 106, 219-232.

Gossler, A. \& Hrabě de Angelis, M. (1998) Somitogenesis. Curr. Top. Dev. Biol., 38, 225 287.

Keynes, R. J. \& Stern, C. D. (1988) Mechanisms of vertebrate segmentation. Development, 103, 413-429.

Kulesa, P. M. \& Fraser, S. E. (2002) Cell dynamics during somite boundary formation revealed by time-lapse analysis. Science, 298, 991-995.

Meinhardt, H. (1982) Models of Biological Pattern Formation. London: Academic.

MeInHardT, H. (1986) Models of segmentation. Somites in Developing Embryos. (R. Bellairs, D. A. Ede \& J. W. Lash, eds). New York: Plenum, pp. 179-189.

Primmett, D. R. N., Norris, W. E., Carlson, G. J., Keynes, R. J. \& Stern, C. D. (1989) Periodic segmental anomalies induced by heat shock in the chick embryo are associated with the cell cycle. Development, 105, 119-130.

Primmett, D. R. N., Stern, C. D. \& Keynes, R. J. (1988) Heat shock causes repeated segmental anomalies in the chick embryo. Development, 104, 331-339.

SAGA, Y. \& TAKEDA, H. (2001) The making of the somite: molecular events in vertebrate segmentation. Nat. Rev. Genet., 2, 835-845.

Sawada, A., Shinya, M., Jiang, Y. J., KaWakami, A., Kuroiwa, A. \& Takeda, H. (2001) Fgf/MAPK signalling is a crucial positional cue in somite boundary formation. Development, 128, 4873-4880. 
SChNell, S. \& MAini, P. K. (2000) Clock and induction model for somitogenesis. Dev. Dyn., 217, 415-420.

Schnell, S., Maini, P. K., Mcinerney, D., Gavaghan, D. J. \& Houston, P. (2002) Models for pattern formation in somitogenesis: a marriage of cellular and molecular biology. C. $R$. Biologies, 325, 179-189.

Schnell, S., Painter, K. J., Maini, P. K. \& Othmer, H. G. (2000) Spatiotemporal pattern formation in early development: a review of primitive streak formation and somitogenesis. Mathematical Models for Biological Pattern Formation. (P. K. Maini \& H. G. Othmer, eds). New York: Springer, pp. 11-37.

Stern, C. D., Fraser, S. E., Keynes, R. J. \& Primmett, D. R. N. (1988) A cell lineage analysis of segmentation in the chick embryo. Development, 104S, 231-244.

Stern, C. D., Hatada, Y., Selleck, M. A. J. \& Storey, K. G. (1992) Relationships between mesoderm induction and the embryonic axes in chick and frog embryos. Development, Supplement, 151-156.

Stickney, H. L., BARresi, M. J. F. \& Devoto, S. H. (2000) Somite development in zebrafish. Dev. Dyn., 219, 287-303.

Stockdale, F. E., Nikovits, JR., W. \& Christ, B. (2000) Molecular and cellular biology of avian somite development. Dev. Dyn., 219, 304-321.

Veini, M. \& Bellairs, R. (1986) Heat shock effects in chick embryos. Somites in Developing Embryos. (R. Bellairs, D. A. Ede \& J. W. Lash, eds). New York: Plenum, pp. 135-145. 